\title{
Rhenium-Dicarbonyl-Nitrosyl-Komplexe mit Imidazol
}

\section{Rhenium Dicarbonyl-Nitrosyl Complexes with Imidazole}

\author{
Dirk Rattat ${ }^{\mathrm{a}, *}$, Alfons Verbruggen ${ }^{\mathrm{a}}$ und Heinz Berke ${ }^{\mathrm{b}}$ \\ ${ }^{a}$ Leuven/Belgien, Labor für radiopharmazeutische Chemie der Katholischen Universität \\ ${ }^{\text {b }}$ Zürich/Schweiz, Anorganisch-chemisches Institut der Universität
}

Bei der Redaktion eingegangen am 28. November 2005.

\begin{abstract}
Different rhenium-dicarbonyl-nitrosyl complexes with imidazole ( $\mathrm{Im})$ as monodentate ligand have been synthesized and characterized, starting from $\left[\mathrm{NEt}_{4}\right]\left[\mathrm{ReCl}_{3}(\mathrm{CO})_{2}(\mathrm{NO})\right]$ and $\left[\operatorname{ReCl}(\mu-\mathrm{Cl})(\mathrm{CO})_{2}(\mathrm{NO})\right]_{2}$. Whereas the complexes $\left[\mathrm{ReCl}_{2}(\mathrm{Im})-\right.$ $\left.(\mathrm{CO})_{2}(\mathrm{NO})\right]$ and $\left[\mathrm{ReCl}(\mathrm{Im})_{2}(\mathrm{CO})_{2}(\mathrm{NO})\right]^{+}$were achieved in high yields, the complex $\left[\operatorname{Re}(\operatorname{Im})_{3}(\mathrm{CO})_{2}(\mathrm{NO})\right]^{2+}$ with three imidazole ligands could only be isolated after complete removal of all halide ions (with $\mathrm{AgBF}_{4}$ ) in low yield. The synthesis of a corresponding
\end{abstract}

${ }^{99 m}$ Tc-dicarbonyl-nitrosyl complex with imidazole opens a new perspective for such compounds as potential radiopharmaceuticals and alternatives to the already established ${ }^{99 \mathrm{~m}} \mathrm{Tc}$-tricarbonyl complexes.

Keywords: Rhenium; Nitrosyl complexes; Carbonyl complexes; Radiochemistry; Technetium; Imidazole

\section{Einleitung}

Der Rhenium-Dicarbonyl-Nitrosyl-Komplex $[\mathrm{ReCl}(\mu-\mathrm{Cl})$ $\left.(\mathrm{CO})_{2}(\mathrm{NO})\right]_{2}$ wurde bereits Anfang der siebziger Jahre beschrieben und seine Synthese später optimiert [1-3]. Damit waren Verbindungen mit einer $\left[\operatorname{Re}(\mathrm{CO})_{2}(\mathrm{NO})\right]^{2+}$ Einheit besser zugänglich, und die Koordination verschiedenartiger Liganden wurde untersucht [4-6]. Die $\left[\mathrm{M}(\mathrm{CO})_{2}(\mathrm{NO})\right]^{2+}-$ Einheit ist isoelektronisch zu der weitaus intensiver erforschten $\left[\operatorname{Re}(\mathrm{CO})_{3}\right]^{+}$-Einheit und deren Technetium-Analog $\left[{ }^{99 \mathrm{~m}} \mathrm{Tc}(\mathrm{CO})_{3}\right]^{+}$. Die entsprechenden $\left[\mathrm{M}(\mathrm{CO})_{2}(\mathrm{NO})\right]$-Komplexe mit radioaktiven Isotopen $(\mathrm{M}=$ ${ }^{186} \mathrm{Re},{ }^{188} \mathrm{Re}$ oder ${ }^{99 \mathrm{~m}} \mathrm{Tc}$ ) sind somit ebenfalls potentielle Kandidaten für medizinisch nutzbare Radiopharmaka (,nitrosyl-approach“) [6]. Von Verbindungen des Typs $\left[\operatorname{ReL}_{3}(\mathrm{CO})_{3}\right]$ und $\left[{ }^{99 m} \mathrm{TcL}_{3}(\mathrm{CO})_{3}\right]\left(\mathrm{L}=\right.$ z.B. Br, Cl, $\mathrm{H}_{2} \mathrm{O}$, $\mathrm{NO}_{3}$ ) ist bekannt, daß die CO-Gruppen unter physiologischen Bedingungen weitgehend inert sind, während die drei anderen monodentaten Liganden in Substitutionsreaktionen relativ leicht ausgetauscht werden können [7, 8]. Dies gilt gleichermaßen für die $\left[\operatorname{Re}(\mathrm{CO})_{2}(\mathrm{NO})\right]^{2+}-$ Einheit, allerdings ändern sich durch die Einführung einer positiv geladenen $\mathrm{NO}^{+}$-Gruppe anstelle einer neutralen $\mathrm{CO}$-Gruppe

\footnotetext{
* Dr. Dirk Rattat

Katholische Universität Leuven

Labor für radiopharmazeutische Chemie

Herestraat 49/O\&N2/8

3000 Leuven/Belgien

Fax: 0032-(0)16-323460

e-mail: dirk.rattat@telenet.be
}

sowohl die Gesamtladung des Komplexes, als auch seine Struktur (durch Aufhebung der Symmetrie). Darüber hinaus wird durch die ausgeprägten $\pi$-Akzeptoreigenschaften des NO-Liganden das Metallzentrum stärker beeinflusst („härteres” Metallzentrum) als bei einem CO-Liganden, und die Position trans-NO gewinnt eine besondere Bedeutung bei der Komplexierung von Liganden (,trans-Effekt") [9]. Das Rhenium-Dicarbonyl-Nitrosyl-System wird in der vorliegenden Studie durch Umsezung mit Imidazol (Im) weiter untersucht, das auch häufig in biologischen Systemenanzutreffen ist, wie z.B. in Purinbasen und Histidin. Imidazol kann als monodentater Ligand an Metallionen koordinieren und bildet besonders mit Übergangsmetallionen erstaunlich stabile Komplexe [10]. Für Rhenium und Technetium sind dagegen nur wenige Komplexe bekannt [11, $12]$.

\section{Ergebnisse und Diskussion}

Als Ausgangsverbindungen zu den Synthesen dienten die Komplexe $\left[\mathrm{NEt}_{4}\right]\left[\mathrm{ReCl}_{3}(\mathrm{CO})_{2}(\mathrm{NO})\right]$ und $\left[\mathrm{NEt}_{4}\right]\left[\mathrm{ReBr}_{3^{-}}\right.$ $\left.(\mathrm{CO})_{2}(\mathrm{NO})\right]$. Ihre Herstellung erfolgte ausgehend von $\left[\mathrm{NEt}_{4}\right]_{2}\left[\mathrm{ReCl}_{3}(\mathrm{CO})_{3}\right], \quad\left[\mathrm{NEt}_{4}\right]_{2}\left[\operatorname{ReBr}_{3}(\mathrm{CO})_{3}\right] \quad$ oder $\left[\mathrm{NEt}_{4}\right]_{2}\left[\mathrm{ReCl}(\mu-\mathrm{Cl})(\mathrm{CO})_{3}\right]_{2}$ in $\mathrm{CH}_{2} \mathrm{Cl}_{2}$ mit $\mathrm{NOBF}_{4}$. [6] Als Alternative zur Umsetzung mit $\mathrm{NOBF}_{4}$ erforschten wir die Verwendung eines Gemisches von $\mathrm{NO}-$ und $\mathrm{NO}_{2}$-Gas. Bei Einleiten dieses Gasgemisches in eine methanolische Lösung von $\left[\mathrm{NEt}_{4}\right]_{2}\left[\operatorname{ReBr}_{3}(\mathrm{CO})_{3}\right]$ ließ sich nach $5 \mathrm{~h}$ ebenfalls der Komplex $\left[\mathrm{NEt}_{4}\right]\left[\mathrm{ReBr}_{3}(\mathrm{CO})_{2}(\mathrm{NO})\right]$ aus der zur Trockne eingeengten Reaktionsmischung mit THF extrahieren.

Bei der Umsetzung der beiden monomeren Rhenium-dicarbonyl-nitrosyl-Komplexe mit einem Äquivalent Imidazol 
(Im) in THF oder Aceton konnte keine Reaktion zu einem Rhenium-Imidazol-Komplex festgestellt werden. Der Ablauf der Reaktion wurde mittels IR-Spektroskopie (Flüssigkeitszelle mit KBr-Fenster oder KBr-Pressling nach Einengen eines Teils der Reaktionslösung) verfolgt. Hierbei wurden ausschließlich die Schwingungsbanden der Ausgangsverbindung detektiert. Die Abspaltung eines $\mathrm{Br}^{-}$-Ions in diesen aprotischen, organischen Lösungsmitteln ist offenbar erschwert. Wurde hingegen die Reaktion mittels ${ }^{1} \mathrm{H}$ NMR in deuteriertem Methanol (oder DMSO) verfolgt, ließ sich die Bildung von Komplexen mit koordiniertem Imidazol beobachten. Bei gleichzeitiger Intensitätsabnahme der Signale von freiem Imidazol bildeten sich neue Signale im Bereich 7,2 bis 8,2 ppm. Nach einer Stunde waren die Signale für freies Imidazol vollständig verschwunden. Dem Hauptprodukt wurden die chemischen Verschiebungen von $8,15,7,54$ und 7,21 ppm zugeordnet, welche den drei Protonen eines koordinierten Imidazols entsprechen. Ein IRSpektrum der eingeengten Reaktionslösung (KBr-Pressling) zeigte Valenzschwingungen für CO (2108 und $\left.2034 \mathrm{~cm}^{-1}\right)$ und NO $\left(1779 \mathrm{~cm}^{-1}\right)$, die sich deutlich von denen der Ausgangsverbindung unterschieden $\left(2093,2018\right.$ und $1771 \mathrm{~cm}^{-1}$ für $\left.\left[\mathrm{NEt}_{4}\right]\left[\mathrm{ReCl}_{3}(\mathrm{CO})_{2}(\mathrm{NO})\right]\right)$.

Zum Vergleich wurde ein Versuch mit dem dinuklearen Komplex $\left[\operatorname{ReCl}(\mu-\mathrm{Cl})(\mathrm{CO})_{2}(\mathrm{NO})\right]_{2}$ durchgeführt, von dem bekannt ist, daß er in koordinierenden Lösungsmitteln wie beispielsweise THF zu einem monomeren Neutralkomplex der Form $\left[\mathrm{ReCl}_{2}(\mathrm{THF})(\mathrm{CO})_{2}(\mathrm{NO})\right]$ aufspaltet $[1,2,4]$. In THF wurde ein Äquivalent Imidazol zugesetzt und quantitativ der Komplex $\left[\operatorname{ReCl}_{2}(\mathrm{Im})(\mathrm{CO})_{2}(\mathrm{NO})\right]$ erhalten. Der Imidazolligand wird in diesem Komplex trans zu einer der Carbonylgruppen koordiniert sein, da die Position transNO aufgrund der besseren "push-pull-Wechselwirkung" von einem Chloroliganden besetzt sein sollte. Als Koordinationsstelle kommt nur die tertiäre Aminfunktion des Imidazols in Frage, da die sekundäre mit ihrem "freien Elektronenpaar" am aromatischen System teilnimmt. Das IRSpektrum (KBr-Pressling) zeigte erneut die Valenzschwingungen für $\mathrm{CO}$ (2108 und $\left.2034 \mathrm{~cm}^{-1}\right)$ und $\mathrm{NO}\left(1779 \mathrm{~cm}^{-1}\right)$, Isomere wurden nicht beobachtet. Im Massenspektrum (MS-EI) zeigte sich der Molekülpeak bei $411 \mathrm{Da}$. Im ${ }^{13} \mathrm{C}$ NMR-Spektrum (in deuteriertem THF) finden sich drei Signale für das koordinierte Imidazol bei 140,4, 130,4 und 118,8 ppm, sowie 188,2 und 187,9 ppm für die beiden Carbonylgruppen. Auch die Elementaranalyse des Reaktionsprodukts bestätigt die Bildung eines Re-Komplexes mit einem Imidazolliganden, wobei das Solvenzmolekül verdrängt wurde. Das Produkt war ein gelber, luftstabiler Feststoff und zeigte auch nach Wochen keinerlei Anzeichen von Zersetzung.

Die Zugabe eines weiteren Äquivalents Imidazol führte unter den gewählten Bedingungen nicht zur Bildung eines Komplexes mit zwei Imidazolliganden. Freies Imidazol war offensichtlich nicht in der Lage in THF einen der beiden verbliebenen Chloroliganden des Komplexes zu verdrängen. Deshalb musste in einem ersten Schritt ein Chloroligand aus dem Komplex entfernt werden. Dies gelang durch eine

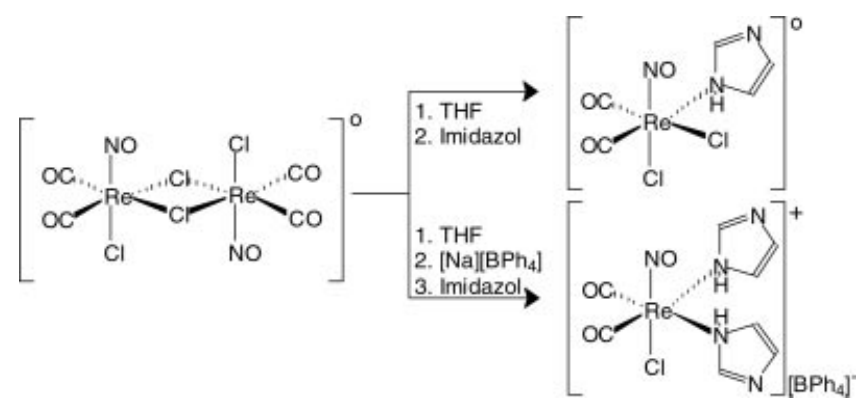

Schema 1 Synthese der Rhenium-dicarbonyl-nitrosyl-Komplexe $\left[\mathrm{ReCl}_{2}(\mathrm{Im})(\mathrm{CO})_{2}(\mathrm{NO})\right]$ und $\left[\mathrm{ReCl}(\mathrm{Im})_{2}(\mathrm{CO})_{2}(\mathrm{NO})\right]\left[\mathrm{BPh}_{4}\right]$ in THF

Umsetzung von $\left[\mathrm{ReCl}(\mu-\mathrm{Cl})(\mathrm{CO})_{2}(\mathrm{NO})\right]_{2}$ mit einem Äquivalent Natriumtetraphenylborat $\left(\mathrm{NaBPh}_{4}\right)$ in THF. Als Zwischenverbindung muss sich dabei der Komplex $\left[\mathrm{ReCl}(\mathrm{Im})(\mathrm{THF})(\mathrm{CO})_{2}(\mathrm{NO})\right]^{+}$gebildet haben, der jedoch nicht isoliert werden konnte. Nach Abfiltrieren des NaClNiederschlages und Zugabe eines Äquivalents Imidazol wurde aus dieser Lösung in guter Ausbeute der Komplex $\left[\mathrm{ReCl}(\mathrm{Im})_{2}(\mathrm{CO})_{2}(\mathrm{NO})\right]\left[\mathrm{BPh}_{4}\right]$ erhalten. Im IR-Spektrum (KBr-Pressling) zeigten sich die entsprechenden CO-Banden bei 2098 und $2020 \mathrm{~cm}^{-1}$ und die NO-Bande bei $1764 \mathrm{~cm}^{-1}$, wobei aufgrund der zusätzlichen elektronenschiebenden Wirkung des zweiten Imidazolliganden gegenüber dem monosubstituierten Komplex niedrigere Wellenzahlen beobachtet wurden. Das Massenspektrum (MSFAB-positiv) zeigte den Molekülpeak bei $444 \mathrm{Da}$ als stärksten Peak, sowie weitere charakteristische Fragmentierungen des Komplexes. ${ }^{1} \mathrm{H}-\mathrm{NMR}-\mathrm{Messungen}$ in deuteriertem Methanol ergaben sich jeweils überlagernde Signale der beiden Imidazolliganden bei 8,13, 7,25 und 7,18 ppm. Das ${ }^{13} \mathrm{C}-\mathrm{NMR}$-Spektrum ergab als chemische Verschiebung 187,9 ppm für die CO-Liganden, sowie 139,6, 129,0 und 117,7 für die Imidazolliganden. Auch die Elementaranalyse bestätigte die Bildung eines Re-Komplexes mit zwei Imidazolliganden. Das Produkt war ebenfalls ein gelber, luftstabiler Feststoff und konnte unzersetzt über Wochen gelagert werden.

$\mathrm{Um}$ in den Komplexen $\left[\mathrm{ReCl}(\mu-\mathrm{Cl})(\mathrm{CO})_{2}(\mathrm{NO})\right]_{2}$ oder $\left[\mathrm{ReCl}(\mathrm{Im})_{2}(\mathrm{CO})_{2}(\mathrm{NO})\right]\left[\mathrm{BPh}_{4}\right]$ einen Chloroliganden aus der Position trans zur Nitrosylgruppe zu entfernen und damit freie Koordinationsstellen für drei Moleküle Imidazol zu schaffen, reichte $\mathrm{NaBPh}_{4}$ in seiner Lewis-sauren abstrahierenden Wirkung nicht aus. Mit Hilfe eines Silbersalzes $\left(\mathrm{AgBF}_{4}\right.$ oder $\left.\mathrm{AgPF}_{6}\right)$ ließ sich jedoch eine vollständige Ausfällung aller Halogenidliganden erreichen. Die folgende Umsetzung mit drei Äquivalenten Imidazol lieferte in niedriger Ausbeute den Komplex $\left[\operatorname{Re}(\operatorname{Im})_{3}(\mathrm{CO})_{2}(\mathrm{NO})\right]^{2+}$. Das Massenspektrum (MS-FAB-positiv) zeigte den erwarteten Molekülpeak bei $476 \mathrm{Da}$. Im Gegensatz zu den vorher beschriebenen Rheniumkomplexen mit einem und zwei Imidazolliganden war dieses Produkt nicht stabil und wurde nicht vollständig charakterisiert.

Eine Übertragung auf wässriges Medium lieferte unterschiedliche Resultate. Bei der Umsetzung von $\left[\mathrm{NEt}_{4}\right][\mathrm{Re}-$ 
$\left.\mathrm{Br}_{3}(\mathrm{CO})_{2}(\mathrm{NO})\right]$ mit einem Äquivalent Imidazol konnte das monosubstituierte Produkt $\left[\operatorname{ReBr}_{2}(\mathrm{Im})(\mathrm{CO})_{2}(\mathrm{NO})\right]$ (zusammen mit nicht umgesetzten Resten der Ausgangsverbindung) nach Einengen der Lösung in THF extrahiert werden. IR-Spektren (KBr-Pressling der erneut eingeengten Lösung) und MS-Spektren (MS-EI) bestätigten die Übereinstimmung mit dem Komplex, der auch in organischen Lösungsmitteln erhalten wurde. Anders als im organischen Medium musste das Imidazol hierbei nicht mit einem Bromoliganden konkurrieren, sondern ein koordiniertes Wassermolekül verdrängen. Die Abspaltung von zwei der drei Halogenidliganden in wässrigen Lösungsmitteln wurde bereits beschrieben [13].

Zur Synthese des Komplexes $\left[\operatorname{ReBr}(\operatorname{Im})_{2}(\mathrm{CO})_{2}(\mathrm{NO})\right]-$ $\left[\mathrm{BF}_{4}\right]$ wurden vor der Umsetzung mit Imidazol aus der Startverbindung zwei Bromoliganden mit $\mathrm{AgBF}_{4}$ entfernt. Erneut zeigten IR- und MS-Spektren eine Übereinstimmung mit dem im organischen Medium erhaltenen Komplex.

Die Isolierung eines Komplexes mit drei Imidazolliganden gelang auch nach Entfernen aller Bromoliganden nicht. Dafür ist das bereits beschriebene Verhalten des $\left[\operatorname{Re}(\mathrm{CO})_{2}(\mathrm{NO})\right]^{2+}-$ Fragments in Wasser verantwortlich $[13,14]$ : Nach vollständigem Entfernen aller Halogenidliganden koordinieren in wässriger Lösung drei Wassermoleküle, von denen eines deprotoniert. Die entstandene Hydroxygruppe kann von Imidazol offensichtlich nicht mehr substituiert werden.

Weiterhin wurde untersucht, ob sich diese Ergebnisse auch auf ${ }^{99 \mathrm{~m}} \mathrm{Tc}$ übertragen lassen, und ob sich die neue Klasse der ${ }^{99 \mathrm{~m}} \mathrm{Tc}-\mathrm{Dicarbonyl-Nitrosyl-Komplexe} \mathrm{prinzipiell}$ für eine radiopharmazeutische Anwendung eignet. Ausgangsmaterial hierzu war immer das Generatoreluat von $\left.{ }^{99} \mathrm{mcO}_{4}\right]^{-}$in $0,9 \%$ iger Kochsalzlösung aus einem handelsüblichen ${ }^{99} \mathrm{Mo} /{ }^{99 m} \mathrm{Tc}-G e n e r a t o r$ (Tyco-Healthcare, Petten, Niederlande), das zum 99mTc-tricarbonyl-Komplex $\left.{ }^{99 \mathrm{~m}} \mathrm{Tc}(\mathrm{CO})_{3}\left(\mathrm{H}_{2} \mathrm{O}\right)_{3}\right]^{+}$umgesetzt wurde [15]. Die Reaktion dieser Technetium-tricarbonyl-Verbindung mit Imidazol lieferte im wässriger Lösung nur ein definiertes Produkt. Die relativ späte HPLC-Retentionszeit von 16,30 min (RP-18 Säule; Gradient Wasser-Acetonitril) weist auf einen lipophilen Komplex hin, in dem Wassermoleküle durch Imidazol ersetzt wurden. Sehr wahrscheinlich handelt es sich dabei um den Neutralkomplex $\left[{ }^{99 \mathrm{~m}} \mathrm{TcCl}(\mathrm{Im})_{2}(\mathrm{CO})_{3}\right]$. Unter ähnlichen Bedingungen ist für Rhenium der vergleichbare Komplex $\left[\operatorname{ReBr}(\operatorname{Im})_{2}(\mathrm{CO})_{3}\right]$ bekannt [11]. Die Verbindung ist ohne Zersetzung über 24 Stunden an Luft stabil. Erhitzen für eine Stunde bei $70{ }^{\circ} \mathrm{C}$ und mit einem Überschuss an Imidazol ändert nicht die Lage des Peaks. Wird die Lösung hingegen mit einem Silbersalz wie $\mathrm{AgNO}_{3}$ versetzt (Chloridfällung), zeigt sich im Chromatogramm nach 10 min Reaktionszeit ein neuer Peak bei einer Retentionszeit von 16,12 min. Dieses Verhalten lässt annehmen, daß sich zuvor noch ein Chloroligand im Komplex befunden hat. Vor dem Hintergrund, daß Komplexe des Typs $\left[\mathrm{MX}_{3}(\mathrm{CO})_{3}\right]^{2-}(\mathrm{M}=$ $\mathrm{Re}, \mathrm{Tc} ; \mathrm{X}=\mathrm{Cl}, \mathrm{Br}$ ) in Wasser alle drei Halogenidliganden unter Bildung eines tris-aquo-Komplexes $\left[\mathrm{M}\left(\mathrm{H}_{2} \mathrm{O}\right)_{3}(\mathrm{CO})_{3}\right]^{+}$
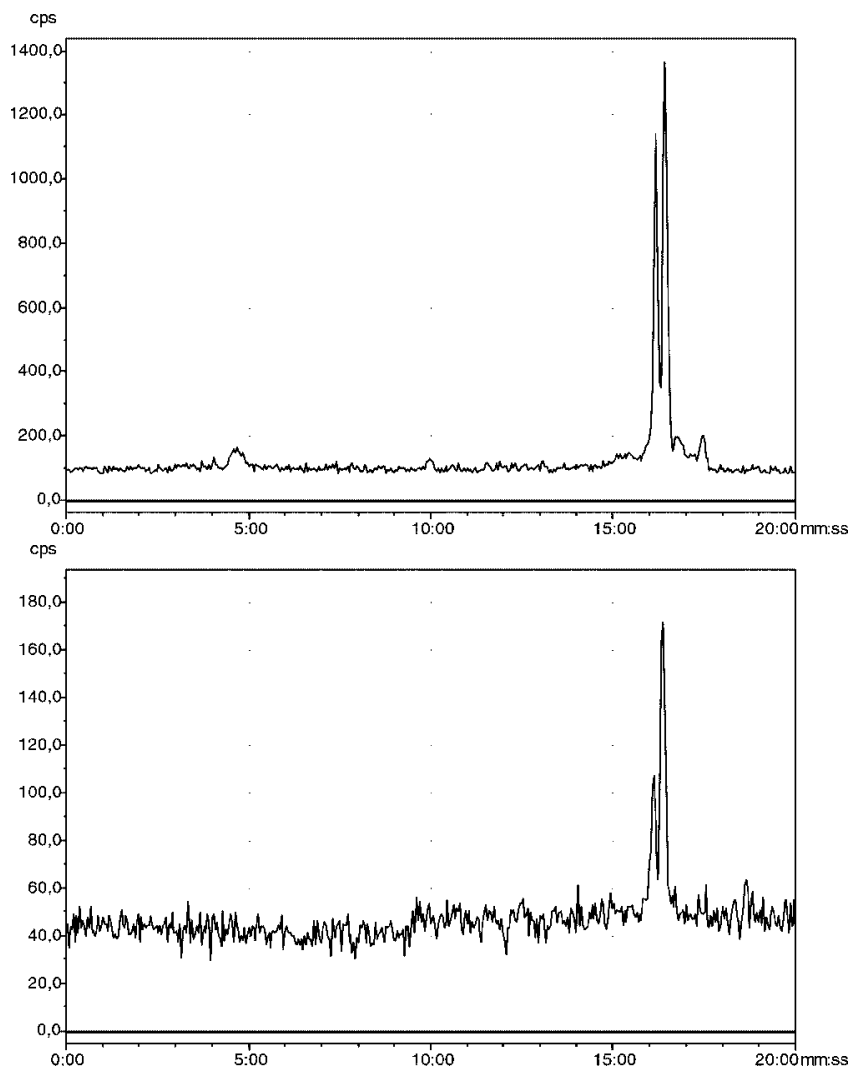

Figur 1 HPLC-Analyse (radiometrisches Signal) des Komplexes $\left[{ }^{99 \mathrm{~m}} \mathrm{TcCl}(\mathrm{Im})_{2}(\mathrm{CO})_{2}(\mathrm{NO})\right]^{+}$. Das Verhältnis der Peaks ändert sich von 45:55 nach $1 \mathrm{~h}$ (oben) auf 34:66 nach $24 \mathrm{~h}$ (unten).

gegen Wasser austauschen [7], ist dieses Ergebnis zumindest ungewöhnlich. Der Peak bei 16,12 min lässt sich auch beobachten, wenn zuerst aus der Precursor-Lösung die Chloridionen mit einem Silbersalz ausgefällt werden (die Chloridionen stammen aus dem Generatoreluat; der ${ }^{99 \mathrm{~m}} \mathrm{Tc}-\mathrm{Cene}$ rator wird mit $0,9 \%$ iger Kochsalzlösung eluiert) und anschließend mit Imidazol umgesetzt wird.

Eine direkte Nitrosylierung der entstandenen ${ }^{99 \mathrm{~m}} \mathrm{Tc}(\mathrm{CO})_{3}$-Verbindung mit $\mathrm{NOBF}_{4}$ in Acetonitril gelang nicht. Demzufolge wurde in einem ersten Schritt die Nitrosylierung durchgeführt, anschließend mit Imidazol umgesetzt. Dazu wurde die wässrige Lösung des Technetiumkomplexes $\left[{ }^{99 \mathrm{~m}} \mathrm{Tc}(\mathrm{CO})_{3}\left(\mathrm{H}_{2} \mathrm{O}\right)_{3}\right]^{+}$mit Tetraethylammoniumchlorid $\left(\mathrm{NEt}_{4} \mathrm{Cl}\right)$ versetzt, die Lösung zur Trockne eingeengt, der Rückstand in Acetonitril aufgenommen und mit $\mathrm{NOBF}_{4}$ zu $\left[{ }^{99 \mathrm{~m}} \mathrm{TcCl}_{3}(\mathrm{CO})_{2}(\mathrm{NO})\right]\left[\mathrm{NEt}_{4}\right]$ umgesetzt.Die Nitrosylierungsreaktion erfolgte damit idealerweise an einem negativ geladenen Komplex [6]. Bei der folgenden Umsetzung mit Imidazol zeigte die HPLC-Analyse nach $1 \mathrm{~h}$ bei $40{ }^{\circ} \mathrm{C}$ zwei Peaks bei einer Retentionszeit von $16,05 \mathrm{~min}$ und 16,20 min (im Verhältnis 45:55). Wurde die Lösung bei Raumtemperatur $12 \mathrm{~h}$ stehengelassen, änderte sich das Verhältnis auf 34:66 zugunsten des späteren Peaks. LC-MSMessungen der Reaktionslösung unterstützen die Annahme, dass es sich bei dem Hauptpeak um den Komplex $\left[{ }^{99 / 99 m} \mathrm{TcCl}(\mathrm{Im})_{2}(\mathrm{CO})_{2}(\mathrm{NO})\right]^{+}$handelt. Der gefundene Wert 
D. Rattat, A. Verbruggen, H. Berke

von 178,589 Da entspricht dem halben berechneten Wert des Molekülpeaks $\left[\mathrm{M}^{+}+\mathrm{H}^{+}\right]^{2+}$ von $178,975 \mathrm{Da}(\mathrm{M} / \mathrm{z} ; \mathrm{z}=$ 2) für einen Rhenium-dicarbonyl-nitrosyl-Komplex mit zwei Imidazol- und einem Chloridliganden (der Molekülpeak $\left[\mathrm{M}^{+}\right]$war nicht zu erkennen).

Zusammenfassend lässt sich sagen, daß Rhenium-dicarbonyl-nitrosyl-Verbindungen mit einem, zwei und drei Imidazolliganden synthetisiert werden konnte, in denen Imidazol ein monodentater Komplexligand ist. Aufgrund der Stabilität der Komplexe mit einem bzw. zwei Imidazolliganden in Wasser, ist prinzipiell ein Einsatz derartiger $\left[\operatorname{Re}(\mathrm{CO})_{2}(\mathrm{NO})\right]^{2+}$ - Verbindungen als Radiopharmaka denkbar (mit den Isotopen ${ }^{186} \mathrm{Re}$ oder ${ }^{188} \mathrm{Re}$ ). Es konnte auch gezeigt werden, daß sich gleichermaßen $\left[{ }^{99 \mathrm{~m}} \mathrm{Tc}(\mathrm{CO})_{2}(\mathrm{NO})\right]^{2+}-$ Komplexe mit monodentaten Liganden herstellen lassen. ${ }^{99 \mathrm{~m}} \mathrm{Tc}$-dicarbonyl-nitrosyl-Verbindungen eröffnen so neue Möglichkeiten bei der Markierung von (Bio-)Molekülen und stellen eine Alternative zu den bereits etablierten ${ }^{99 \mathrm{~m}} \mathrm{Tc}$-Tricarbonyl-Verbindungen dar.

\section{Experimenteller Teil}

Die Synthesen der Metallkomplexe wurden unter Normalbedingungen ohne besondere Vorsichtsmaßnahmen bezüglich Luft- oder Lichtempfindlichkeit durchgeführt.

Imidazol ist kommerziell erhältlich (Acros, Belgien).

Die IR-Spektren wurden mit einem Bio-Rad FTS-45 Spektrometer (BIO-RAD, Hercules, USA) bzw. Perkin-Elmer FT-IR-16PC Spektrometer (Perkin-Elmer, Wellesley, USA) aufgenommen. Die NMR-Spektren wurden auf einem Varian Gemini 2000 (300 MHz) Spektrometer mit einer 4n-Sonde aufgenommen (Varian, Palo Alto, USA). Im Falle der ${ }^{1} \mathrm{H}$ - und ${ }^{13} \mathrm{C}-\mathrm{NMR}$-Spektren diente jeweils die chemische Verschiebung $\delta$ des deuterierten Lösungsmittels relativ zu TMS als interner Standard. Die Massenspektren (MS-EI und MS-FAB) wurden auf einem Finnigan/MAT-8320 (MS) aufgenommen (Thermo Electron Corporation/Finnigan, San Jose, USA), bei FAB-Spektren diente 3-Nitrobenzylakohol als Matrix. Es sind nur die $m / z$-Werte charakteristischer Ionen angegeben (unkorrigierte relative Häufigkeiten in Klammern). Die Elementaranalysen wurden mit einem LECO CHNS-932 Analysegerät durchgeführt (LECO, St.Joseph, USA).

Die ${ }^{99 m}$ Tc-Komplexe wurden mittels "reversed phase HPLC" analysiert (XTerra RP-18 Säule $4.6 \mathrm{~mm}$ x $250 \mathrm{~mm}$; Waters, Brüssel, Belgien; Gradient von $0.1 \%$ Trifluoressigsäure in Wasser nach $0.1 \%$ Trifluoressigsäure in Acetonitril in 20 min; Flussgeschwindigkeit $1 \mathrm{ml} / \mathrm{min}$ ).

Die Bestimmung der molekularen Masse erfolgte mittels LC-MS (Waters Separation Module, XTerra MS C18 Säule $50 \mathrm{~mm} \mathrm{x}$ $2.1 \mathrm{~mm}$, 3-Inch NaI(Tl) Detektor, Micromass LCT Massenspektrometer und MassLynx Software, Waters-Micromass, Manchester, UK). Die Säule wurde bei einer Flussrate von $300 \mu \mathrm{l} / \mathrm{min}$ eluiert (Wasser-Acetonitril, linearer Gradient von 0\% Acetonitril nach $50 \%$ Acetonitril in $20 \mathrm{~min}$ ). Zur Bestimmung der exakten Masse wurde eine 0.01 \%ige Lösung von Kryptofix 2.2.2 in $\mathrm{CH}_{3} \mathrm{CN} / \mathrm{H}_{2} \mathrm{O}$ (1:1) als Referenz zugesetzt (Flussrate $1 \mu 1 / \mathrm{min}$ ).

Synthese von $\left[\mathbf{R e C l}_{\mathbf{2}}(\mathbf{I m})(\mathbf{C O})_{2}(\mathbf{N O})\right]$. Dazu wurde eine Lösung von $200 \mathrm{mg}(0,29 \mathrm{mmol})\left[\mathrm{ReCl}(\mu-\mathrm{Cl})(\mathrm{CO})_{2}(\mathrm{NO})\right]_{2}$ in $10 \mathrm{ml}$ THF (abs.) mit 39,5 mg (0,58 mmol, 1 eq pro Re) Imidazol versetzt. Nach $1 \mathrm{~h}$ bei Raumtemperatur wurde die klare, gelbe Lösung zur Trockne eingeengt und der Rückstand mit einer Vakuumpumpe getrocknet. Nach Umkristallisieren aus $\mathrm{CH}_{2} \mathrm{Cl}_{2} /$ Diethylether wurden $218 \mathrm{mg}$ $(0,53 \mathrm{mmol}, 92 \%)\left[\mathrm{ReCl}_{2}(\mathrm{Im})(\mathrm{CO})_{2}(\mathrm{NO})\right]$ als gelbes Pulver isoliert. Alternative Synthese von $\left.\left[\mathbf{R e C l}_{\mathbf{2}}(\mathbf{I m})(\mathbf{C O})_{\mathbf{2}} \mathbf{( N O}\right)\right]$ in Wasser: $50 \mathrm{mg}$ $(0,081 \mathrm{mmol})$ des Komplexes $\left[\mathrm{NEt}_{4}\right]\left[\mathrm{ReCl}_{3}(\mathrm{CO})_{2}(\mathrm{NO})\right]$ wurden in $4 \mathrm{ml}$ Wasser gelöst, mit 5,5 mg (0,081 mmol, 1 eq pro Re) Imidazol versetzt und $1 \mathrm{~h}$ bei Raumtemperatur gerührt. Nach Einengen zur Trockne wurde das Produkt aus dem Rückstand mit THF extrahiert. (Anmerkung: Nicht umgesetztes Ausgangsmaterial wird ebenfalls extrahiert, weshalb die oben beschriebene Synthese in THF effektiver ist.)

IR $\left(\mathrm{CH}_{2} \mathrm{Cl}_{2} ; \mathrm{cm}^{-1}\right): v(\mathrm{CO}) 2108 \mathrm{vs}, v(\mathrm{CO}) 2034$ vs, $v$ (NO) 1779 vs. MS (EI): $411\left(\mathrm{M}^{+} ; 18 \%\right), 383\left(\mathrm{M}^{+}\right.$- $\left.\mathrm{CO} ; 97 \%\right), 355\left(\mathrm{M}^{+}\right.$- $\left.2 \mathrm{CO} ; 100 \%\right), 325$ $\left(\mathrm{M}^{+}-2 \mathrm{CO}-\mathrm{NO} ; 77 \%\right), 272\left(\operatorname{Re}(\mathrm{CO})_{2}(\mathrm{NO}) ; 23 \%\right), 68$ (Imidazol; $\left.33 \%\right)$. ${ }^{1} \mathrm{H}-\mathrm{NMR}\left(\mathrm{d}^{6}\right.$-DMSO): $\delta[\mathrm{ppm}]=8,15(\mathrm{~m}, 1 \mathrm{H}, \mathrm{H} 2) ; 7,54(\mu, 1 \mathrm{H}, \mathrm{H} 4) ; 7,21$ (m, 1H, H5). ${ }^{13}$ C-NMR (d $\left.{ }^{4}-\mathrm{THF}\right): \delta[\mathrm{ppm}]=188,2(2 \mathrm{CO}), 140,4$ (Im-C2), 130,4 (Im-C5), 118,8 (Im-C4). Elementaranalyse: $\mathrm{C}_{5} \mathrm{Cl}_{2} \mathrm{H}_{4} \mathrm{~N}_{3} \mathrm{O}_{3} \mathrm{Re}(411.22)$ C, 14,60; H, 0,98; N, 10,22. Exp.: C, 15,28; H, 1,21; N, 10,06\%.

Synthese von $\left[\mathbf{B P h}_{\mathbf{4}}\right]\left[\mathbf{R e C l}(\mathbf{I m})_{\mathbf{2}}(\mathbf{C O})_{\mathbf{2}}(\mathbf{N O})\right]$. Dazu wurde eine Lösung von $200 \mathrm{mg}(0,29 \mathrm{mmol})\left[\mathrm{ReCl}(\mu-\mathrm{Cl})(\mathrm{CO})_{2}(\mathrm{NO})\right]_{2}$ in $10 \mathrm{ml}$ THF (abs.) mit 198,5 mg (0,58 mmol, 1 eq pro Re) $\mathrm{NaBPh}_{4}$ versetzt. Nach 1-2 h bei RT unter kräftigem Rühren trübte sich die Lösung, über Nacht fiel feinkristallines $\mathrm{NaCl}$ aus. Die filtrierte gelbe Lösung wurde mit $79 \mathrm{mg}(1,16 \mathrm{mmol}, 2$ eq pro Re) Imidazol versetzt, bei Raumtemperatur über Nacht gerührt und anschlieBend zur Trockne eingeengt. $435 \mathrm{mg}(0,57 \mathrm{mmol}, 98 \%)\left[\mathrm{BPh}_{4}\right][\mathrm{Re}-$ $\left.\mathrm{Cl}(\mathrm{Im})_{2}(\mathrm{CO})_{2}(\mathrm{NO})\right]$ verblieben als gelber, kristalliner Feststoff.

Alternative Synthese von $\left[\mathbf{R e C l}(\mathbf{I m})_{2}(\mathbf{C O})_{2}(\mathbf{N O})\right]^{+}$in Wasser: $50 \mathrm{mg}$ $(0,081 \mathrm{mmol})$ des Komplexes $\left[\mathrm{NEt}_{4}\right]\left[\mathrm{ReCl}_{3}(\mathrm{CO})_{2}(\mathrm{NO})\right]$ wurden in $4 \mathrm{ml}$ Wasser gelöst, mit $31,5 \mathrm{mg}(0,162 \mathrm{mmol}, 2$ eq pro Re) AgBF4 versetzt und 15 min bei Raumtemperatur gerührt. Nach Abfiltrieren des AgCl-Niederschlages wurden 5,5 mg (0,081 mmol, 1 eq pro $\mathrm{Re})$ Imidazol zugegeben und $1 \mathrm{~h}$ bei Raumtemperatur gerührt. Nach Einengen zur Trockne wurde der Komplex $\left[\mathrm{BF}_{4}\right]\left[\mathrm{ReCl}(\mathrm{Im})_{2^{-}}\right.$ $\left.(\mathrm{CO})_{2}(\mathrm{NO})\right]$ aus dem Rückstand mit THF extrahiert.

IR $\left(\mathrm{CH}_{2} \mathrm{Cl}_{2} ; \mathrm{cm}^{-1}\right): v$ (CO) 2098 vs, $v$ (CO) 2020 vs, $v$ (NO) 1764 vs. MS (FAB ${ }^{+}$, Aceton): $444\left(\mathrm{M}^{+} ; 48 \%\right), 416\left(\mathrm{M}^{+}-\mathrm{CO} ; 10 \%\right), 307(\mathrm{ReCl}$ $\left.(\mathrm{CO})_{2}(\mathrm{NO}) ; 22 \%\right), 289\left(\mathrm{ReCl}\left(\mathrm{C}_{3} \mathrm{H}_{4} \mathrm{~N}_{2} ; 18 \%\right) .{ }^{1} \mathrm{H}-\mathrm{NMR}\left(\mathrm{CD}_{3} \mathrm{OD}\right): \delta\right.$ $[\mathrm{ppm}]=8,13\left(\mathrm{~m}, 2 \mathrm{H}, \mathrm{H} 2\right.$ und $\left.\mathrm{H} 2^{\prime}\right) ; 7,25\left(\mathrm{~m}, 2 \mathrm{H}, \mathrm{H} 4\right.$ und $\left.\mathrm{H} 4^{\prime}\right) ; 7,18(\mathrm{~m}$, 2H, H5 und $\left.\mathrm{H}^{\prime}\right),{ }^{13} \mathrm{C}-\mathrm{NMR}\left(\mathrm{d}^{4}-\mathrm{THF}\right): \delta[\mathrm{ppm}]=187,9(2 \mathrm{CO}), 139,6$ (Im-C2), 129,0 (Im-C5), 117,7 (Im-C4). Elementaranalyse: $\mathrm{BC}_{32} \mathrm{ClH}_{28} \mathrm{~N}_{5} \mathrm{O}_{3} \operatorname{Re}(763,08) ; \mathrm{C}, 50,37 ; \mathrm{H}, 3,70 ; \mathrm{N}, 9,18$. Exp.: C, 49,38; H, 3,$98 ; \mathrm{N}, 9,43 \%$

Synthese von $\left.{ }^{{ }^{99 m}} \mathbf{T c}(\mathbf{C O})_{3}\left(\mathbf{H}_{2} \mathbf{O}\right)_{3}\right]^{+}$. Der ${ }^{99 m}$ Tc-tricarbonyl-Komplex wurde nach einer Literaturvorschrift erhalten [15].

Synthese von $\left.{ }^{99 m} \mathbf{T c C l}(\mathbf{I m})_{2}(\mathbf{C O})_{3}\right] . \quad 0,3 \mathrm{ml}$ einer Lösung von $\left[{ }^{99 \mathrm{~m}} \mathrm{Tc}(\mathrm{CO})_{3}\left(\mathrm{H}_{2} \mathrm{O}\right)_{3}\right]^{+}$wurden mit einer Spatelspitze Imidazol versetzt und der $\mathrm{pH}$-Wert mit $1 \mathrm{~N} \mathrm{NaOH}$ auf 10 eingestellt. Nach einer Reaktionszeit von $20 \mathrm{~min}$ bei $70^{\circ} \mathrm{C}$ wurde die Reaktionslösung mittels HPLC analysiert. Das Chromatogramm zeigte eine quantitative Umsetzung zum gewünschten Produkt bei einer Retentionszeit von $16,30 \mathrm{~min}$.

Synthese von $\left[^{{ }^{99 m}} \mathbf{T c C l}(\mathbf{I m})_{2}(\mathbf{C O})_{2}(\mathbf{N O})\right]^{+}$. 0,3 $\mathrm{ml}$ einer frisch hergestellten Lösung von $\left[{ }^{99 \mathrm{~m}} \mathrm{Tc}(\mathrm{CO})_{3}\left(\mathrm{H}_{2} \mathrm{O}\right)_{3}\right]^{+}$wurden mit einer Spatelspitze $\mathrm{NEt}_{4} \mathrm{Cl}$ versetzt und die Reaktionslösung bei $40{ }^{\circ} \mathrm{C}$ im Stickstoff-Gegenstrom zur Trockne eingeengt (verschlossenes $5 \mathrm{ml}$ Spitzvial). Der verbleibende weiße Rückstand wurde mit $150 \mu 1$ Acetonitril und einer Spatelspitze $\mathrm{NOBF}_{4}$ versetzt, die Lösung mit $\mathrm{N}_{2}$ begast und über Nacht bei Raumtemperatur gerührt. Ein 
HPLC-Chromatogramm der Reaktionslösung zeigte, dass $75 \%$ des ${ }^{99 \mathrm{~m}} \mathrm{Tc}(\mathrm{CO})_{3}{ }^{+}$nitrosyliert worden waren $\left(25 \%{ }^{99 \mathrm{~m}} \mathrm{TcO}_{4}{ }^{-}\right)$. Nicht umgesetztes $\mathrm{NOBF}_{4}$ wurde abgetrennt, die Reaktionslösung mit einer Spatelspitze Imidazol versetzt und eine Stunde bei $40{ }^{\circ} \mathrm{C}$ gerührt. Eine Analyse mittels HPLC zeigte zwei neue radioaktive Peaks bei $R_{t}=16,05$ min und $R_{t}=16,20$ min im Verhältnis 45:55. Wurde die Reaktionslösung bei Raumtemperatur über Nacht stehengelassen, änderte sich das Verhältnis auf 34:66 zugunsten des späteren Peaks.

LC-MS $\left(\mathrm{ES}^{+}\right)$: 178,589 Da (theoretisch: 178,975 Da) entspricht dem halben berechneten Wert des Molekülpeaks $\left[\mathrm{M}^{+}+\mathrm{H}^{+}\right]^{2+}$ $(m / z$ für $\mathrm{z}=2)$ für einen Rhenium-dicarbonyl-nitrosyl-Komplex mit zwei Imidazol- und einem Chloridliganden.

\section{Literatur}

[1] F. Zingales, A. Trovati, P. Cariati, P. Uguagliati, Inorg. Chem. 1971, 10, 507.

[2] F. Zingales, A. Trovati, P. Uguagliati, Inorg. Chem. 1971, 10, 510.

[3] H.-U. Hund, U. Ruppli, H. Berke, Helv. Chim. Acta 1993, 76, 963.
[4] D. Veghini, S. E. Nefedov, H. Schmalle, H. Berke, J. Organomet. Chem. 1996, 526, 117; R. Urban, D. Veghini, H. Berke, W. Beck, Z. Anorg. Allg. Chem. 2005, 631, 2715-2718.

[5] D. Rattat, P. A. Schubiger, H. G. Berke, H. Schmalle, R. Alberto, Cancer Biother. Radio. 2001, 16, 339.

[6] D. Rattat, A. Verbruggen, H. Schmalle, H. G. Berke, R. Alberto, Tetrahedron Lett. 2004, 45, 4089.

[7] R. Alberto, R. Schibli, R. Waibel, U. Abram, A. P. Schubiger, Coord. Chem. Rev. 1999, 190-192, 901.

[8] R. Schibli, A. P. Schubiger, Eur. J. Nucl. Med. 2002, 29, 1529.

[9] G. B. Richter-Addo, P. Legzdins, „Metal Nitrosyls“, Oxford University Press, New York, Oxford, 1992.

[10] L. E. Kapinos, B. Song, H. Sigel, Inorg. Chim. Acta 1998 , $280,50$.

[11] R. Alberto, Habilitationsschrift, Universität Zürich 1996.

[12] P. H. Fackler, M. J. Lindsay, M. J. Clarke, Inorg. Chim. Acta 1985, 109, 39

[13] D. Rattat, A. Verbruggen, H. G. Berke, R. Alberto, J. Organomet. Chem. 2004, 689, 4833 .

[14] P. Kurz, D. Rattat, D. Angst, H. Schmalle, B. Spingler, R. Alberto, H. Berke, W. Beck, Dalton Trans. 2005, 21, 804-810.

[15] R. Alberto, R. Schibli, A. Egli, A. P. Schubiger, U. Abram, T. A. Kaden, J. Am. Chem. Soc. 1998, 120, 7987. 